\section{Magnetic yeast}

Manipulating either culture conditions or genes can precipitate iron particles in yeast cells and render them magnetic.

Yeast are not necessarily known for their magnetic personalities (although some scientists might disagree). Migrating birds, monarch butterflies and other types of migratory or homing animals, by contrast, perform some of the more awe-inspiring feats in the animal world, moving in a precise and directional fashion across vast distances in a process that involves sensing the Earth's magnetic field. Magneto-sensing depends in part on the presence of magnetic particles in the bodies of these organisms. Now, in recently published work, Keiji Nishida and Pam Silver at Harvard Medical School show a proof of concept that laboratory strains of yeast, too, can be magnetized.

Simply culturing yeast in high concentrations of iron salts, the researchers observed, resulted in a fraction (about half) of the cells becoming magnetic. Mutation of the vacuolar iron transporter Ccclp and cytosolic expression of the three genes encoding human ferritin, an iron-sequestering protein, could additionally increase the number of magnetic cells. Upon examination under an electron microscope, the researchers observed electron-dense particles of varying size and shape, which they could show contained iron, in cells cultured under conditions that induced magnetization.

One of the attractive properties of yeast is its tractability as a genetic model; Nishida and Silver made use of the available yeast knockout collection to probe for genes that affect magnetization. They first tested 60 candidate genes implicated in iron homeostasis or oxidative stress and found that TCO89, which encodes a nonessential component of the target-of-rapamycin complex TORC1, results in reduced magnetization when mutated. The effect is independent of vacuolar sequestration, requires TORC1 activity and correlates with changes in redox state of the cells. In a more expanded candidate screen, the researchers identified genes involved in redox regulation and carbon metabolism that also reduce yeast magnetization when mutated.

Whether or not yeast can more systematically be used as a genetic system to study the formation of biomagnetic particles will depend, Silver points out, on whether this system reflects natural processes. "We know so little about biomagnetism," she says, "but it's interesting that when we look for genes that affect it even in this engineered system, we get some highly conserved genes." Those might be candidates worth testing in other cell types, she suggests.

More practically, if magnetization could be induced efficiently in strains of yeast that are typically used for bioprocessing-largescale protein production, for instance-then magnetic capture could be used as a separation method for cells that produce, but do not secrete, a protein of interest. "Obviously the process would need a lot of optimization before anyone could do that," Silver says. However, at least for the laboratory strains of yeast, the cells can be efficiently captured on magnetic columns.

It should be noted that magnetic sensing in animals does not depend only on magnetic particles; specialized magnetic fieldsensing photoreceptors are also thought to be involved. Even if the yeast system turns out to be informative for the formation of physiological magnetic deposits, therefore, it is unlikely to provide insight into many aspects of magnetic sensing (the magnetosensitive behavior of Drosophila melanogaster, reported a few years ago, may make for a more promising genetic model in this regard). However, cells as diverse as bacteria and the neurons of humans with neurodegenerative disease are known to accumulate iron deposits; a simple and genetically tractable system, such as this one in yeast, could provide useful hints into both natural and pathogenic aspects of this process.

\section{Natalie de Souza}

\section{RESEARCH PAPERS}

Nishida, K. \& Silver, P.A. Induction of biogenic magnetization and redox control by a component of the target of rapamycin complex 1 signaling pathway. PLoS Biol. 10, e1001269 (2012). 